\title{
Early stage phase separation in ternary alloys: A test of continuum simulations
}

\author{
S. O. Poulsen*, P. W. Voorhees \\ Department of Materials Science and Engineering, Northwestern University, Evanston, IL, \\ 60208, USA
}

\begin{abstract}
Phase separation in the $\gamma-\gamma^{\prime}$ alloy $5.2 \% \mathrm{Al}-14.2 \% \mathrm{Cr}-\mathrm{Ni}$ at $873.15 \mathrm{~K}$ has been investigated by three-dimensional phase-field simulations, employing a model where both thermodynamic and kinetic parameters were experimentally verified. 510 individual nuclei corresponding to a density of $10^{24} \mathrm{~m}^{-3}$ were introduced in accordance with classical nucleation theory, and the microstructural evolution was simulated up to a time of $32 \mathrm{~h}$. The microstructural evolution was characterized, and regimes were identified according to the dominant mechanisms for microstructural evolution, where the final regime was found to be a coarsening regime obeying well-known power laws for the particle density and the average $\gamma^{\prime}$ particle radius. The evolution of the volume-averaged composition of the particles was found to follow a complicated trajectory, while the composition of individual particles were found to depart significantly from the average. The simulations were compared to experimental results from the literature based on atom probe tomography, and in general good qualitative correspondence was found, albeit with quantitative differences. These are discussed in terms of the assumptions inherent to the phase-field model, and by extension to most common continuum models of diffusive phase transformations.
\end{abstract}

Keywords: Phase field modeling, Ni-Al-Cr, Phase separation, Coalescence, Coarsening

\footnotetext{
${ }^{*}$ Corresponding author

Email address: stefan.poulsen@northwestern.edu (P. W. Voorhees)
}

Preprint submitted to Acta Materialia

April 25, 2016 


\section{Introduction}

It is well-known that the microstructure of metals and alloys are intimately linked with the mechanical performance of these materials. In the case of $\mathrm{Ni}$ based superalloys which are used extensively in high-temperature conditions 5 such as in gas turbine engines, the beneficial properties are mainly derived from $\mathrm{L} 1_{2}$-ordered $\gamma^{\prime}$ particles that are nearly coherent with the disordered $\gamma$ matrix phase [1]. Predicting and controlling the dual-phase microstructure of Ni-based superalloys through the alloy composition and the processing path is therefore of great technological importance.

Such a dual-phase microstructure may be precipitated from solid solution by quenching a homogenized $\gamma$ phase ingot into the $\gamma-\gamma^{\prime}$ phase field and aging, during which $\gamma^{\prime}$ particles nucleate, grow into the supersaturated matrix, and eventually coarsen.

This process has been extensively studied experimentally by Atom Probe

15 Tomography (APT), which is a destructive three-dimensional method, so dynamics must be inferred from "snapshots" of microstructures at different aging times. Quantitative simulations of the dynamics of the phase separation process is therefore an attractive tool to rationalize and interpret experimental results, and ultimately to guide future experiments as part of the Integrated Computational Materials Engineering (ICME) approach to materials development $[2,3]$.

Several studies combining experimental investigations with simulations, especially Lattice Kinetic Monte Carlo (LKMC) methods, may be found in the literature, e.g. $[4,5,6]$. The LKMC framework is particularly suited to investigations of $\gamma-\gamma^{\prime}$ phase separation in $\mathrm{Al}-\mathrm{Cr}-\mathrm{Ni}$ alloys due to the negligible misfit between small $\gamma^{\prime}$ particles and the $\gamma$ matrix. In this inherently atomistic method, vacancy-mediated diffusion is governed by short-range atomic interactions. Therefore the scales of the problems that may be solved with LKMC is independent of the feature size of the microstructure. 
On the other hand, early-stage precipitation dynamics are commonly predicted with classical models, employing classical nucleation theory combined with diffusion equations in the continuum limit, e.g. using the popular software packages PrecipiCalc by QuesTek Innovations LLC, or TC-PRISMA by Thermo-Calc Software AB. These approaches rely on the validity of classical nucleation theory and certain assumptions in going to the continuum limit, the most notable being a permanent equilibrium distribution of vacancies throughout the material, and these assumptions are rarely questioned.

In this paper, we investigate phase separation dynamics in the Ni-based $\gamma-\gamma^{\prime}$ alloy $5.2 \% \mathrm{Al}-14.2 \% \mathrm{Cr}-\mathrm{Ni}$ during aging at $873.15 \mathrm{~K}$ with three-dimensional phase-field simulations. The phase-field model was coupled to a CALPHAD40 optimized thermodynamics database, and CALPHAD-style optimized atomic mobility assessments. Thus, both thermodynamics and kinetics are considered experimentally verified in the continuum limit. 510 identical nuclei chosen in accordance with the predictions of classical nucleation theory corresponding to an initial $\gamma^{\prime}$ particle density of $10^{24} \mathrm{~m}^{-3}$ were introduced (thus corresponding 45 to the case of site saturated nucleation) in a random spatial arrangement, and the microstructural evolution was simulated up to a time of $32 \mathrm{~h}$. We take the investigated alloy to be an appropriate model system for Ni-based superalloys, as it contains the most common alloying elements of these.

We present our findings, and compare these to the experimental results pub50 lished by Booth-Morrison et al. 7] produced by APT on a comparable alloy (6.5\% $\mathrm{Al}-9.5 \% \mathrm{Cr}-\mathrm{Ni})$ aged at the same temperature. As the phase-field simulations are based on a continuum model, we may examine the validity of the assumptions inherent to these models, and we discuss the similarities and differences between experiment and simulation in terms of the limitations of the 55 model employed. 


\section{Phase-field model description}

\subsection{Phase-field variables and energetics}

In phase-field models of microstructural evolution, the microstructural features of interest are described by sets of phase-field variables. These variables are defined in the entire system, and are required to vary smoothly in both time and space. Bulk regions are characterized by the phase-field variables attaining some set of equilibrium values, and interfacial regions are characterized by intermediate values, which occur over a specific non-zero width. The reader is referred to e.g. the text by Provatas and Elder [8], and the reviews by Chen [9], Moelans et al. [10], and Steinbach [11] for comprehensive introductions to the phase-field method.

In the present work, we describe the local composition of the Al-Cr-Ni alloy by the two molar fraction variables $\vec{x}=\left(x_{\mathrm{Al}}, x_{\mathrm{Cr}}\right)$ and so $x_{\mathrm{Ni}}=1-x_{\mathrm{Al}}-x_{\mathrm{Cr}}$. The phase structure is described by two phase-field variables $\vec{\eta}=\left(\eta_{\gamma}, \eta_{\gamma^{\prime}}\right)$. As we do not require these to sum to unity, we cannot interpret these unambiguously as phase fractions, however, we employ the re-scaling of the original phase-field variables introduced by Moelans [12]

$$
\vec{\phi}=\left(\phi_{\gamma}, \phi_{\gamma^{\prime}}\right)=\left(\frac{\eta_{\gamma}^{2}}{\eta_{\gamma}^{2}+\eta_{\gamma^{\prime}}^{2}}, \frac{\eta_{\gamma^{\prime}}^{2}}{\eta_{\gamma}^{2}+\eta_{\gamma^{\prime}}^{2}}\right)
$$

which sums to unity everywhere, and may thus be regarded as phase fractions. The phase-field variables $\vec{\eta}$ are mathematical constructs, with no intrinsic physical interpretation. Note that using only one phase-field variable for the $\gamma^{\prime}$ phase means that $\gamma^{\prime}-\gamma^{\prime}$ anti-phase boundaries are not possible.

Furthermore, we employ four phase composition variables $\vec{x}_{\gamma}=\left(x_{\mathrm{Al}}^{\gamma}, x_{\mathrm{Cr}}^{\gamma}\right)$ and $\vec{x}_{\gamma^{\prime}}=\left(x_{\mathrm{Al}}^{\gamma^{\prime}}, x_{\mathrm{Cr}}^{\gamma^{\prime}}\right)$, which may be interpreted as describing the local composition in the respective phases, and the weighted sum of these are therefore required to equal the local composition [13]

$$
\vec{x}=\sum_{\rho=\gamma, \gamma^{\prime}} \phi_{\rho} \vec{x}_{\rho}
$$


These quantities will be elaborated on in Sec. 2.2. For an isothermal, isobaric system, the convenient thermodynamic potential is the Gibbs free energy $F$, which we take to be given by

$$
F=\int\left(f_{\text {int }}+f_{\text {bulk }}\right) d V
$$

where $f_{\text {int }}$ is the contribution from interfaces

$$
f_{\mathrm{int}}=m f_{0}+\frac{\kappa}{2} \sum_{\rho=\gamma, \gamma^{\prime}}\left|\nabla \eta_{\rho}\right|^{2}
$$

where $m$ is a model parameter, $\kappa$ is the gradient energy coefficient, and $f_{0}$ is the double-well

$$
f_{0}=\sum_{\rho=\gamma, \gamma^{\prime}}\left[\frac{\eta_{\rho}^{4}}{4}-\frac{\eta_{\rho}^{2}}{2}\right]+\frac{\delta}{2} \eta_{\gamma}^{2} \eta_{\gamma^{\prime}}^{2}+\frac{1}{4}
$$

with $\delta$ being a model parameter. The functional $f_{\text {bulk }}$ of Eq. (3) is the contribution of the chemical composition to the Gibbs free energy $F$, which we take to be given by the expression by Eiken et al. [13]

$$
f_{\mathrm{bulk}}=\sum_{\rho=\gamma, \gamma^{\prime}} \phi_{\rho} f_{\rho}\left(\vec{x}_{\rho}\right)+\sum_{k=\mathrm{Al}, \mathrm{Cr}} \tilde{\mu}_{k}\left(x_{k}-\sum_{\rho=\gamma, \gamma^{\prime}} \phi_{\rho} x_{\rho k}\right)
$$

where the $f_{\rho}$ are the free energy densities of the individual bulk phases, and the quantities $\overrightarrow{\tilde{\mu}}=\left(\tilde{\mu}_{\mathrm{Al}}, \tilde{\mu}_{\mathrm{Cr}}\right)$ are Lagrange multipliers which enforce Eq. (2). The model neglects elastic energy, and is therefore limited to small particle sizes, where this effect is negligible, and the precipitates remain unfaceted, as is observed to be the case for particle sizes up to at least $R \sim 10 \mathrm{~nm}$ by experiment [7].

\subsection{Phase composition}

The phase compositions $\vec{x}_{\rho}$ and Lagrange multipliers $\overrightarrow{\tilde{\mu}}$ are determined from the local microstructural configuration by minimization of the bulk free energy Eq. (6), with respect to $\vec{x}_{\rho}$ and $\overrightarrow{\tilde{\mu}}$. The minimal energy configuration will be a stationary point of Eq. (6), and will therefore fulfill

$$
\frac{\partial f_{\gamma}}{\partial x_{k}}=\frac{\partial f_{\gamma^{\prime}}}{\partial x_{k}}=\tilde{\mu}_{k}(k=\mathrm{Al}, \mathrm{Cr})
$$


which is the well-known parallel tangent assumption. We can identify $\overrightarrow{\tilde{\mu}}$ as interdiffusion potentials, which in the present case of Ni considered the dependent component are related to the chemical potentials through $\overrightarrow{\tilde{\mu}}=\left(\mu_{\mathrm{Al}}-\mu_{\mathrm{Ni}}, \mu_{\mathrm{Cr}}-\mu_{\mathrm{Ni}}\right)$ and so the requirement of minimal bulk free energy is equivalent to requiring equal interdiffusion potentials in the interfacial regions of the two phases. The equality of the diffusion potentials is commonly referred to as quasi-equilibrium [13], since it does not imply thermodynamic equilibrium, but only local equilib-

85 rium of the phase compositions of the coexisting phases.

For an equilibrium interface, the Gibbs free energy $F$ will also be minimized with respect to the phase structure variables $\vec{\eta}$, and therefore the phase fractions $\vec{\phi}$. The conditions for this minimum are mathematically identical to the wellknown common tangent plane construction, as employed during construction of 90 phase diagrams [14]. So for an equilibrium interface, the phase compositions of the locally coexisting phases $\vec{x}_{\rho}$ will be constant, and equal to their respective tie line compositions, while the local composition $\vec{x}$ will trace the tie line through the interface.

The quasi-equilibrium condition complicates calculations, since the phase compositions and diffusion potentials must be determined in all interfacial regions in the system, however, the quasi-equilibrium condition is chosen to cause the decoupling of bulk and interfacial energies, and so allows the interfacial widths to be chosen as convenient to the problem at hand, independent of the free energy functions $f_{\rho}[13,12]$, which may be a great convenience.

\subsection{Kinetic equations}

The rate of change of the composition is taken to obey an "Onsager-like" relationship with the driving force for diffusion given by the diffusion potential $[12]$

$$
\frac{\partial x_{k}}{\partial t}=\nabla \cdot\left(\sum_{l=\mathrm{Al}, \mathrm{Cr}} \mathbf{M}_{k l} \nabla \tilde{\mu}_{l}\right) \quad(k=\mathrm{Al}, \mathrm{Cr})
$$


where $\mathbf{M}$ is a matrix of interdiffusion mobilities in the volume-centered frame of reference, which we assume may be approximated by interpolation

$$
\mathbf{M}=\phi_{\gamma} \mathbf{M}^{\gamma}(\vec{x})+\phi_{\gamma^{\prime}} \mathbf{M}^{\gamma^{\prime}}(\vec{x})
$$

and so the mobility matrices are evaluated at the local composition, rather than the phase composition.

The rate of change of the phase structure is taken to obey a time-dependent Ginzburg-Landau equation

$$
\frac{\partial \eta_{\rho}}{\partial t}=-L\left(m \frac{\partial f_{0}}{\partial \eta_{\rho}}-\kappa \nabla^{2} \eta_{\rho}+\frac{\partial f_{\mathrm{bulk}}}{\partial \eta_{\rho}}\right)\left(\rho=\gamma, \gamma^{\prime}\right)
$$

Here, $L$ is a mobility parameter, which in the case of bulk diffusion controlled transformations is related to the chemical mobility at the interface, as shown by $\operatorname{Kim}[15]$

$$
L=L_{0}\left(\sum_{k=\mathrm{Al}, \mathrm{Cr}}\left(x_{\gamma k}^{\mathrm{eq}}-x_{\gamma^{\prime} k}^{\mathrm{eq}}\right) \sum_{l=\mathrm{Al}, \mathrm{Cr}}\left[\mathbf{M}^{-1}\right]_{k l}\left(x_{\gamma l}^{\mathrm{eq}}-x_{\gamma^{\prime} l}^{\mathrm{eq}}\right)\right)^{-1}
$$

$L_{0}$ is a model parameter, the superscript eq denotes the tie line compositions of the interface, and $\mathbf{M}^{-1}$ is the inverse of the mobility matrix $\mathbf{M}=$

${ }_{105} \frac{1}{2}\left(\mathbf{M}^{\gamma}+\mathbf{M}^{\gamma^{\prime}}\right)$, which has been shown by Moelans [12] to result in negligible solute trapping at the relatively slowly migrating interfaces occurring during growth and coarsening in the solid state.

\subsection{Free energies and mobility parameters}

It is common in the literature to employ parabolic free energy densities, which may have been fitted to CALPHAD-optimized free energy functions. This is desirable as parabolic free energy functions result in models that are easily analyzed and computationally simple, but depending on the problem, the parabolic approximation may not be sufficiently precise.

In the present work, we have chosen a more general approach, namely directly employing CALPHAD-optimized free energy densities as assessed by Saunders [16]. This assessment employs a two-sublattice model for the ordered 
$\gamma^{\prime}$ phase [17], so the free energy density is a function of the a priori unknown site fractions. In a recent work, Zhang et al. [18] describes a method to directly incorporate sublattice models into phase-field simulations, where the sublattices exchange mass locally at a finite rate. However, the benefits of adopting this method for the current problem were not clear, so an infinite rate of exchange between the sublattices was assumed in the current work so the sublattice occupation is chosen everywhere as the minimal energy configuration. Details on the numeric implementation are presented in Sec. 2.6.

For the required interdiffusional mobilities, we employ the CALPHAD-style optimized assessments by Engström and Ågren for the $\gamma$ phase [19], and by Campbell for the $\gamma^{\prime}$ phase [20], which gives diagonal atomic mobility matrices in the lattice-fixed frame of reference. The atomic mobilities in the respective phases are converted to interdiffusion mobilities in the volume-fixed frame of reference by the transformation [21]

$$
M_{k l}^{\rho}=V_{m} \sum_{n=\mathrm{Al}, \mathrm{Cr}, \mathrm{Ni}}\left(\delta_{l n}-x_{l}\right)\left(\delta_{k n}-x_{k}\right) x_{n} \Omega_{n}^{\rho}
$$


The spatial differential operators were discretized with five-point central finite-difference stencils, and the temporal differential operator was discretized with the forward Euler method.

The grid spacing was chosen to be $h=1.1 \AA$ (the choice of this specific value will be explained in Sec. 3) and the time step was chosen to be $\Delta t=10^{-2} \mathrm{~s}$.

Periodic boundary conditions were imposed, and the simulations were executed in parallel on 200 cores of Northwestern University's Quest High Performance Computing Cluster.

\subsection{Computational implementation and validation}

Prior to simulation, the free energy densities are determined on a fine (in this case $1000 \times 1000)$ grid over the range $x_{\mathrm{Al}}+x_{\mathrm{Cr}} \leq 1$. In the case of the ordered $\gamma^{\prime}$ phase, this requires constrained numeric optimization to determine site fractions as functions of local composition. Parabolas are fitted to a local neighborhood of all grid points, and the polynomial coefficients are stored for both phases, which allows the local morphology (functional value, gradient and Hessian matrix) of the free energy densities to be determined by interpolation of the polynomial coefficients. This allows solution of the quasi-equilibrium condition iteratively by Newton's method for constrained optimization, which is elaborated upon in the appendix.

We note that 1) this approach may give inaccurate results for systems with very low concentrations of one or more components (which is irrelevant for current work), as the parabolic fit cannot accurately reproduce the divergence of the derivatives of the free energy density due to the entropic contribution [24], and 2) the memory requirements for this scheme scales linearly with the number of phases, but exponentially with the number of chemical components, thus for simulating higher-order systems i.e. quatenary or higher, other schemes should be considered, such as the one recently proposed by Larsson and Höglund [25].

Solution of the quasi-equilibrium condition is the main computational bottleneck. Efficiency has been increased by 1) applying knowledge about the structure of the linear system of equations that must be solved in every itera- 
tion of Newton's method, and 2) only solving the quasi-equilibrium condition at positions where the local cumulative change over time (sum of the absolute values of all phase-field variables) exceeded a value $\epsilon$. For the current work, a value of $\epsilon=10^{-5}$ was found to introduce negligible error by comparison to simulations with a value of $\epsilon=0$, while providing a significant speed up.

The interdiffusional mobilities were fully dependent on composition, and in the case of the $\gamma^{\prime}$ phase on the site fraction. The mobilities were determined on a grid, as was the case for the polynomial coefficients for the free energy functions, and retrieved by interpolation during simulation.

The computational implementation and the chosen parameters was validated by simulating growth of a planar interface, the position of which was found to follow the well-known $t^{1 / 2}$ law, and by simulating coarsening of two $\gamma^{\prime}$ particles in a $\gamma$ matrix, where the particle radii were found to be parabolic in time as expected [26]. No grid-oriented anisotropy due to poor spatial discretization was observed.

\section{Initial conditions}

We generate initial conditions for the simulations by introducing a random spatial arrangement of identical $\gamma^{\prime}$ nuclei into a $\gamma$ matrix of uniform composition, thus modeling a site saturated nucleation process. For a classical model such as the one employed in current work, the critical nucleus of classical nucleation theory gives the nucleus size below which the nucleus will dissolve, and above which the nucleus will grow into the supersaturated matrix. The nucleus radius $R^{*}$ and composition $\vec{x}_{\gamma^{\prime}}$ is determined by

$$
\mu_{k}^{\gamma}\left(\vec{x}_{\gamma}\right)=\mu_{k}^{\gamma^{\prime}}\left(\vec{x}_{\gamma^{\prime}}\right)+\frac{2 \sigma V_{m}}{R^{*}},(k=\mathrm{Al}, \mathrm{Cr}, \mathrm{Ni})
$$

As this introduces a non-zero volume fraction of $\gamma^{\prime}$ into the $\gamma$ matrix, we further require that

$$
\vec{x}_{\text {alloy }}=\phi_{\gamma^{\prime}} \vec{x}_{\gamma^{\prime}}+\left(1-\phi_{\gamma^{\prime}}\right) \vec{x}_{\gamma}
$$

where $\phi_{\gamma^{\prime}}=n V^{*}$ is the volume fraction of $\gamma^{\prime}$ particles, $n$ is the desired number density, and $V^{*}$ is the volume of the critical nucleus. Eqs. (13) and (14) 
was solved iteratively by relaxation to determine the critical radius $R^{*}$, and compositions of the matrix and nuclei, $\vec{x}_{\gamma}$ and $\vec{x}_{\gamma^{\prime}}$.

For the alloy composition $5.2 \% \mathrm{Al}-14.2 \% \mathrm{Cr}-\mathrm{Ni}$, and a number density of $1.02 \times 10^{24} \mathrm{~m}^{-3}$, we find a critical radius of $R^{*}=5.77 \AA$, a nucleus composition 190 of $17.36 \% \mathrm{Al}-7.728 \% \mathrm{Cr}-\mathrm{Ni}$ and a matrix composition of $5.190 \% \mathrm{Al}-14.21 \% \mathrm{Cr}-\mathrm{Ni}$ by employing the CALPHAD-optimized free energies to determine the required chemical potentials. These (excluding the initial matrix composition which is virtually indistinguishable from the alloy composition) are indicated on a partial phase diagram in Fig. 1. We note that the composition of the critical nucleus is not on the tie line, as predicted by Philippe et al. [27].

The critical nucleus size $R^{*}$ represents the smallest length scale of interest in the system, and so to resolve this, the $\gamma-\gamma^{\prime}$ interface width $W$ must be chosen no greater. Additionally, the smallest value of the grid spacing which was found to not introduce numeric artifacts was chosen. A result of this is that the critical nucleus size $R^{*}$ marks the size below which $\gamma^{\prime}$ particles will dissolve into the matrix independent of the matrix composition.

We introduce the nuclei with constant composition and sharp interfaces, with the smallest size greater than $R^{*}$ allowed by the spatial discretization.

A recent approach to nucleation in phase-field models by Jokisaari et al. ${ }_{205}[28]$ introduces nuclei into a system by introducing a seed exclusively in the order parameter of the nucleating phase, which then provides a driving force for "self-assembly" of precipitates in the usual kinetic equations of the system, resulting in a nucleation current that respects the global conservation laws. Such an approach is not applicable in the current system, as the $\gamma^{\prime}$ phase is found to be unstable at the matrix composition in the current work. Therefore, a seed introduced in the order parameter in the current $\gamma-\gamma^{\prime}$ system will not lead to "self-assembly" of precipitates, but will rather rapidly transform to the matrix phase. We note that this complication may be avoided by using parabolic approximations to the free energy, as the thermodynamic stability is then constant in composition. 


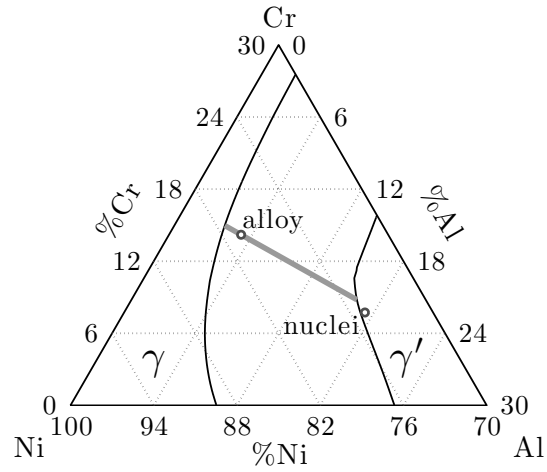

Figure 1: Partial phase diagram of the $\gamma-\gamma^{\prime}$ system with the alloy composition, resulting tie line, and composition of the critical nucleus indicated.

\section{Simulation results}

Three sets of simulations were executed on a grid with $500^{3}$ grid points corresponding to a cube with side length $55 \mathrm{~nm}$, with a total of $520 \gamma^{\prime}$ particles for times up to $32 \mathrm{~h}$ at a temperature of $873.15 \mathrm{~K}$. In the following, the presented average quantities are the average of the three simulations, and an initial transient related to the sharp interface initial conditions of $10 \mathrm{~min}$ is omitted.

\subsection{Characterization of microstructural evolution}

An example of a simulated microstructure at three different times is shown in Fig. 2. At the earliest time shown (a, 1h10min), the $\gamma^{\prime}$ particles are largely identical, due to the choice of initial conditions. For the middle time (b, 3h32min), some particles exhibit non-spherical shapes due to coalescence with one or more other particles. No particles smaller than the particles in the earliest time shown are observed. At the latest time shown (c, 19h00min) the non-spherical particles from the middle time have largely regained a spherical shape, and several particles smaller than the particles observed at the earliest time may be seen.

The volume fraction of $\gamma^{\prime}$ particles shown in Fig 3 increases monotonically towards the thermodynamic equilibrium volume fraction of $\phi_{0}=12.6 \%$ consistent with the CALPHAD-optimized free energies applied in current work. The 

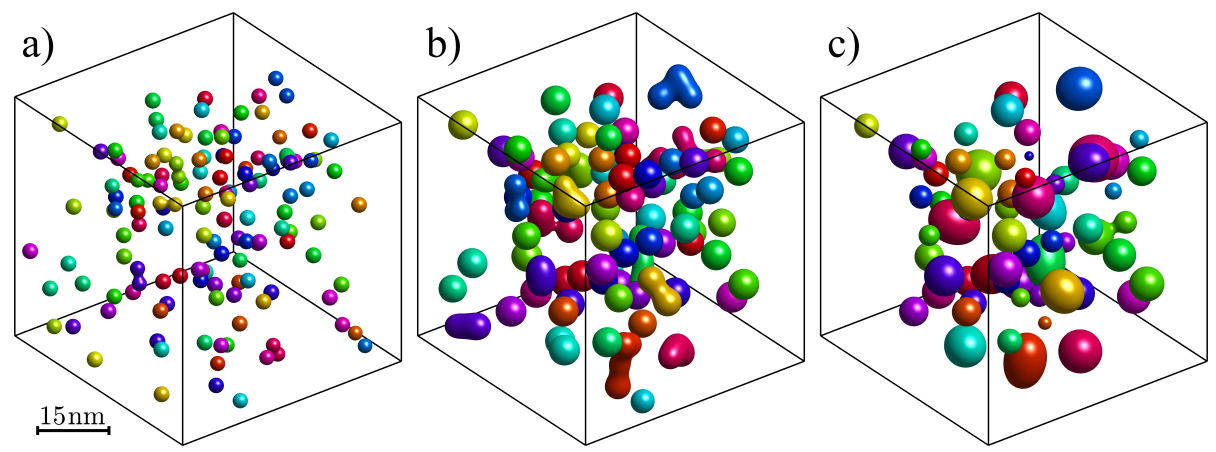

Figure 2: Simulated evolution of $\gamma^{\prime}$ particles in a $\gamma$ matrix for the alloy $5.2 \% \mathrm{Al}-14.2 \% \mathrm{Cr}-\mathrm{Ni}$ (at.\%) aged at $873.15 \mathrm{~K}$ at aging times a) $1 \mathrm{~h} 10 \mathrm{~min}$. b) $3 \mathrm{~h} 32 \mathrm{~min}$. c) $19 \mathrm{~h} 00 \mathrm{~min}$. Visualized by the $\phi_{\gamma^{\prime}}=\frac{1}{2}$ surfaces. Particles intersecting the periodic boundaries not shown. Colors are arbitrary.

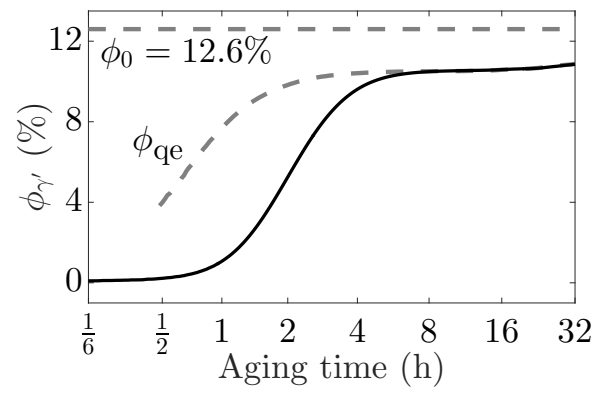

Figure 3: $\gamma^{\prime}$ volume fraction, equilibrium volume fraction $\phi_{0}$, and quasi-equilibrium volume fraction $\phi_{\mathrm{qe}}$.

transformation is seen to slow down significantly when the volume fraction is $\sim 2 \%$ lower than $\phi_{0}$.

This discrepancy is found to be a result of capillarity: Assuming the system is undergoing diffusive coarsening, the critical radius $R_{c}$ below which particles shrink and above which particles grow, is equal to the average particle size, $R_{c}=\langle R\rangle[26]$, and the critical particle fulfills Eqs. (13) (with $R_{c}=R^{*}$ ). These equations have been solved numerically subject to Eq. (14), to give the relationship between quasi-equilibrium volume fraction $\phi_{\mathrm{qe}}$ and critical radius $R_{c}$. We then relate $\phi_{\mathrm{qe}}$ to time by assuming that $R_{c}=\langle R\rangle$, and using the values of $\langle R(t)\rangle$ from the simulations to determine the predicted $\phi_{\mathrm{qe}}(t)$. This is shown 
in Fig. 3. The good agreement after a time of $t \approx 8 \mathrm{~h}$ implies that growth has ceased, and the microstructural evolution is dominated by coarsening. Further increases in volume fraction towards the thermodynamic equilibrium value are therefore the result of an increasing average particle size, rather than growth into a supersaturated matrix, which is seen to progress at a much slower rate.

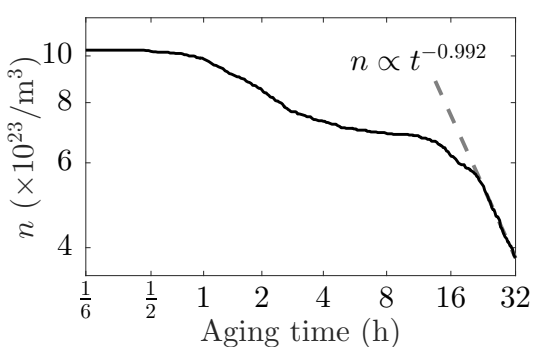

(a)

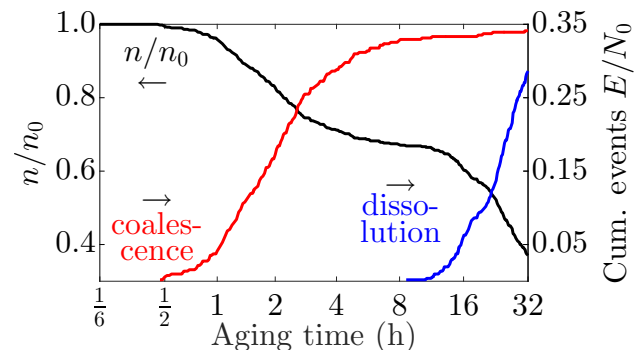

(b)

Figure 4: a) Density of $\gamma^{\prime}$ particles. b) Particle elimination events decomposed into coalescence - and dissolution events (red and blue lines, right axis) and relative density of $\gamma^{\prime}$ particles overlaid for convenience (black line, left axis).

Fig. 4a shows the time evolution of the $\gamma^{\prime}$ particle density. The particle density begins decreasing at a time of $t \approx \frac{1}{2} \mathrm{~h}$, slows down considerably at a time of $t \approx 3 \mathrm{~h}$, before speeding up around $t \approx 12 \mathrm{~h}$, and finally reaching the well-known power law relationship $n \propto t^{m}$ at $t \approx 22 \mathrm{~h} 37 \mathrm{~m}$, where $m=-0.992 \approx-1$ is determined by least-squares. The characteristic shape of the particle density curve may be understood when considering the two mechanisms which may be responsible for particle elimination in the current model: Coalescence, where particles in contact join to form a single larger particle, and dissolution of small particles into the matrix. The cumulative number of events relative to the initial particle number are shown in Fig. 4b, where it is seen that the first decrease in the particle density $\left(t \approx \frac{1}{2} \mathrm{~h}\right)$ is due to coalescence events, and the subsequent speed up $(t \approx 12 \mathrm{~h})$ is largely due to the onset of dissolution events. This is consistent with the early microstructural evolution dominated by growth into the supersaturated matrix (average interparticle distance is decreasing, high probability of 
coalescence) and the late stages dominated by coarsening (large particles grow, small particles shrink so average interparticle distance is increasing, thus low probability of coalescence.)

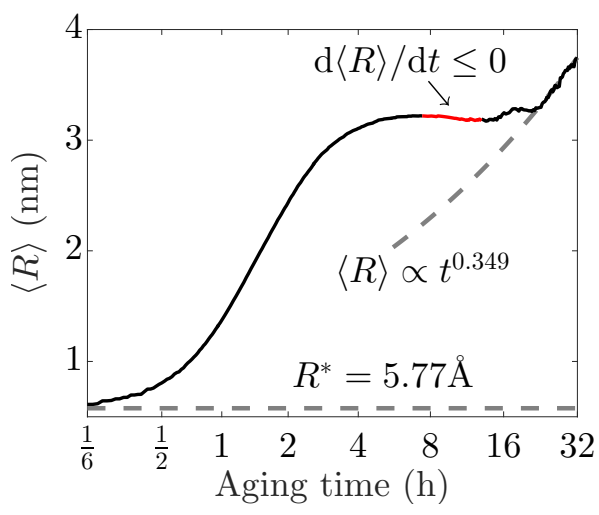

(a)

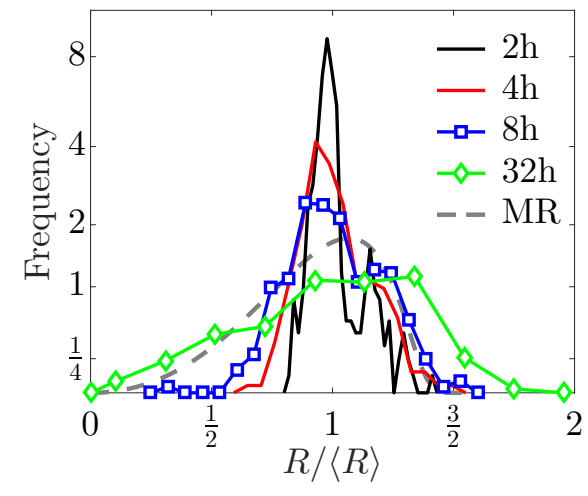

(b)

Figure 5: a) Average particle radius. Red line indicates negative growth rate. b) Particle size distributions (PSDs) at various times. Also shown is the Marqusee-Ross PSD for an equilibrium volume fraction $\phi_{0}=12.6 \%$ [29]. The number of bins is chosen by the method of Shimazaki and Shinomoto [30] to reduce bias in each instance.

The average $\gamma^{\prime}$ particle radius is shown in Fig. 5a. It is seen to largely resemble the evolution of the $\gamma^{\prime}$ volume fraction shown in Fig. 3 until a time of $t \approx 4 \mathrm{~h}$. However, in contrast to the non-decreasing $\gamma^{\prime}$ volume fraction, the average particle radius is seen to decrease between times $t=7 \mathrm{~h} 24 \mathrm{~min}$ and $t=12 \mathrm{~h} 52 \mathrm{~min}$, before again increasing, and reaching the power law relationship $\langle R\rangle \propto t^{m}$ at $t \approx 22 \mathrm{~h} 37 \mathrm{~m}$, with $m=0.349 \approx 1 / 3$ determined by least-squares. Furthermore, the theory for coarsening in multicomponent alloys by Morral and Purdy [31] gives an expression for the rate constant $K$ of the relationship $\langle R\rangle^{3} \propto$ $K t$ which for the current system is evaluated to be $K=2.10 \times 10^{-31} \mathrm{~m}^{3} \mathrm{~s}^{-1}$ 275 (Morral and Purdy) while the power law fit to the simulation results gives $K=2.04 \times 10^{-31} \mathrm{~m}^{3} \mathrm{~s}^{-1}$ (current work) giving a deviation of less than $3 \%$, thus verifying that the phase-field model is accurately reproducing the predictions of a model based upon a sharp interface analysis. 
The counterintuitive reduction in average $\gamma^{\prime}$ particle radius as a function of decrease or altogether eliminate this effect, as smaller particles nucleated late in the growth stage would dissolve more readily.

We may now summarize the observed microstructural evolution by subdivi- 
the three-dimensional case (while remembering that the critical radius for nucleation in a two-dimensional system is half that of the three-dimensional system) gave results that were both quantitatively and qualitatively different from the three-dimensional results: The transformation occurs at a much increased rate 335 (likely due to the longer reaching logarithmic diffusion fields around particles in two dimensions compared to the $r^{-1}$ fields in three dimensions), and the microstructural evolution does not follow the six regimes characterized above. Thus, we conclude that for systems with diffusional interactions, conclusions about the microstructural evolution of three-dimensional systems drawn on the 


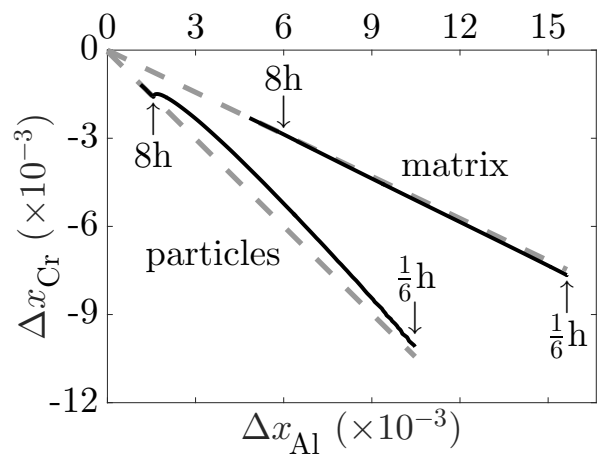

Figure 6: Trajectories of volume-averaged matrix composition and volume-averaged particle composition minus corresponding equilibrium (tie line) value. Gray lines are straight lines least-squares fitted to points for times $t \geq 8 \mathrm{~h}$.

basis of two-dimensional calculations must be scrutinized carefully.

\subsection{Evolution of particle and matrix composition}

In order to examine the composition of the $\gamma^{\prime}$ particles and the $\gamma$ matrix, we take points as belonging to a particle if $\phi_{\gamma^{\prime}} \geq 0.995$, belonging to the matrix if $\phi_{\gamma} \geq 0.995$, and consequently belonging to an interfacial region elsewhere. The specific threshold was chosen based on inspection of the simulation results to minimize the volume of the interfacial regions while not introducing significant bias in the composition averaged over individual particles.

Fig. 6 shows the trajectory of the volume-averaged composition of the particles and the matrix, as the difference between the composition and the corresponding equilibrium (tie line) compositions. The gray lines shown are straight lines fitted to points for times $t \geq 8 \mathrm{~h}$, and both are found to approach the tie line value, and so it is possible that the gray lines would provide accurate extrapolation for times $t>32 \mathrm{~h}$. The trajectories become well-described by straight lines around the time when coarsening becomes the dominant mechanism for microstructural evolution, consistent with the theory by Philippe and Voorhees [32]. Before $8 \mathrm{~h}$ the evolution of the matrix composition is well-approximated by a straight line, which is almost parallel to the extrapolated line, while the tra- 
jectory of the volume-averaged particle composition is more complicated with a non-monotonic $\mathrm{Cr}$ content, and a kink in the curve at a time of $8 \mathrm{~h}$.

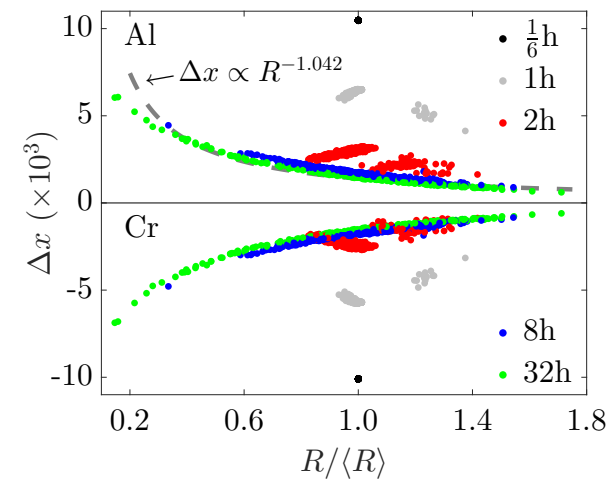

(a)

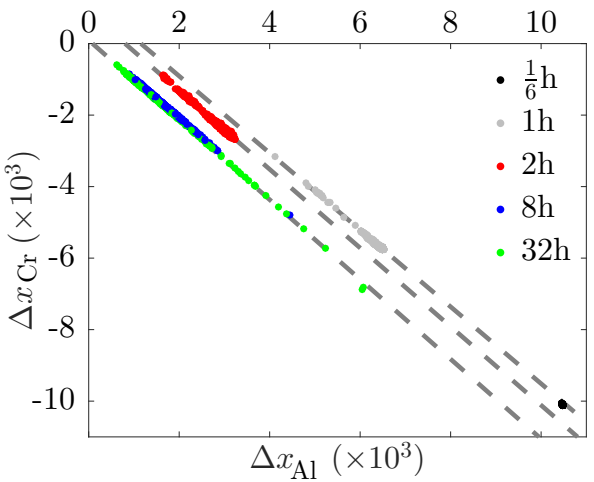

(b)

Figure 7: Volume-averaged composition of individual $\gamma^{\prime}$ particles minus equilibrium (tie line) value. a) As a function of normalized particle radius for various times. Also shown is a power law fit to $x_{\mathrm{Al}}$ for time $t=32 \mathrm{~h}$. b) Cr content of individual $\gamma^{\prime}$ particles as function of $\mathrm{Al}$ content. Gray lines are straight lines least-squares fitted to times $t=1 \mathrm{~h}, 2 \mathrm{~h}$, and $32 \mathrm{~h}$ (parameters in text body).

Turning attention to the volume-averaged composition of individual $\gamma^{\prime}$ particles, Fig. 7a shows composition as a function of normalized particle radius for various times. For $t=\frac{1}{6} \mathrm{~h}$, the particles are virtually identical due to the initial conditions of the simulations. At $t=1 \mathrm{~h}$, the particles are found in three clusters, reflecting the number of coalescence events undergone (zero, one or two), and this clustering is still evident at $t=2 \mathrm{~h}$, but not at the later times shown. At $t=8 \mathrm{~h}$ and $t=32 \mathrm{~h}$, the composition of the particles resemble a continuum. During coarsening, the theory by Philippe and Voorhees [32] predicts a $R^{-1}$ dependence in the particle composition, however, although the distribution of particle compositions at $t=32 \mathrm{~h}$ visually resemble a $R^{-1}$ dependence, and least-squares fitting a power law relationships $\Delta x \propto R^{m}$ finds $m=-1.042$ (shown on Fig. 7a), but this is found to be inaccurate for particles smaller than approximately $R<0.4\langle R\rangle$. This is explained by the finite diffusivity in the $\gamma^{\prime}$ particles, that above-mentioned theory does not account for: The small 
particles are shrinking at high radial rates, and therefore are unable to maintain described by a straight line. This finding is in agreement with the theory of Philippe and Voorhees [32].

Finally, Fig. 8 shows the evolution of the composition of five individual 


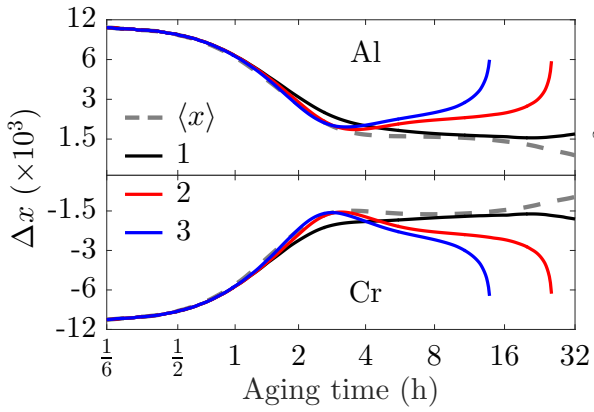

(a)

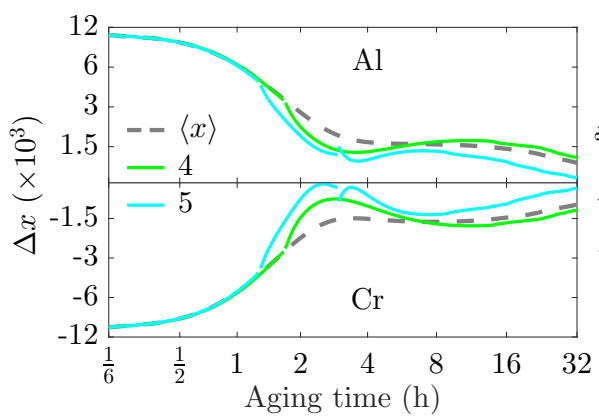

(c)

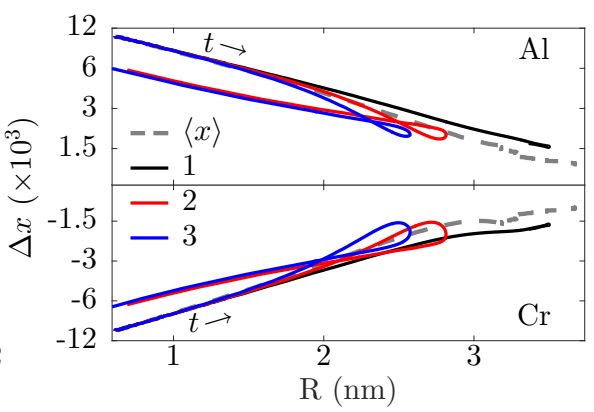

(b)

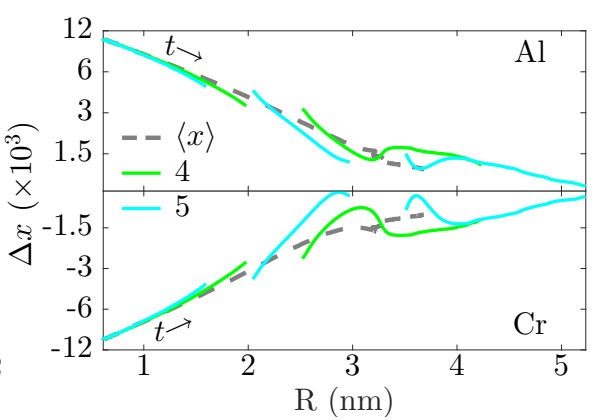

(d)

Figure 8: Composition of five individual $\gamma^{\prime}$ particles minus equilibrium (tie line) composition. $\mathrm{a}$ and c) As a function of aging time. b and d) As a function of particle radius. Gray lines are the volume-averaged particle composition, in Figs. $8 \mathrm{~b}$ and $8 \mathrm{~d}$ as a function of average particle radius $\langle R\rangle$. Note that particles 4 and 5 experienced coalescence events, which results in discontinuities in their composition.

particles with the volume-averaged particle composition shown in gray for comparison as a function of time (Figs. 8a and 8c) and as a function of particle radius (Figs. $8 \mathrm{~b}$ and $8 \mathrm{~d}$ ). If the system was a binary alloy, the compositions would only be functions of the particle size. Since this is not necessarily the case in a ternary alloy we therefore investigate the magnitude of this effect of $\mathrm{Al}-\mathrm{Cr}-\mathrm{Ni}$ alloys at the early stages of precipitation.

Particle 1 (black curves in Figs. $8 \mathrm{a}$ and $8 \mathrm{~b}$ ) reaches its maximal size at a time of $t=17 \mathrm{~h} 40 \mathrm{~min}$ before shrinking, however its composition is found to approach its equilibrium value until a time of $t=20 \mathrm{~h} 52 \mathrm{~min}$ where the particles compo- 
sition (all components simultaneously) begins departing from the equilibrium value.

Particles 2 and 3 (red and blue curves in Figs. 8a and 8b) are found to increase in size until times $t=5 \mathrm{~h} 00 \mathrm{~min}$ and $t=4 \mathrm{~h} 04 \mathrm{~min}$ before shrinking and eventually dissolving. As opposed to particle 1, their extrema in composition do not occur simultaneously: For particle 2, the $\mathrm{Al}$ composition approaches its equilibrium value until a time of $t=3 \mathrm{~h} 36 \mathrm{~min}$ before departing, while this occurs for the Cr composition at $t=3 \mathrm{~h} 08 \mathrm{~min}$, and for particle 3 the corresponding times are $t=3 \mathrm{~h} 16 \mathrm{~min}$ and $t=2 \mathrm{~h} 50 \mathrm{~min}$. This results in compositional trajectories vs. particle size that loops so the curves crosses themselves. These loops are smaller in the case of $\mathrm{Al}$ than in the case of $\mathrm{Cr}$, and we note that this is not possible in a binary alloy, since then concentration $\propto 1 / R$.

Particles 1-3 initially follow the volume-averaged particle composition (gray curves) before monotonically departing away from the equilibrium composition.

Particle 4 (green curves in Figs. 8c and 8d) experiences a coalescence event at $t=1 \mathrm{~h} 40 \mathrm{~min}$. This does not alter the composition appreciably, as the coalescing particles are found to be almost identical in both size and composition: Particle size increases by a factor of $\Delta R=1.27$ compared to $\Delta R=2^{1 / 3}=1.26$ as expected for identical particles, and the composition changes by factors $\Delta x_{\mathrm{Al}}=0.96$ and $\Delta x_{\mathrm{Cr}}=0.95$. The particle size increases monotonically, but the composition shows local extrema: A locally closest approach to the equilibrium value is found at $t=3 \mathrm{~h} 32 \mathrm{~min}$ for $\mathrm{Al}$ and $t=2 \mathrm{~h} 52 \mathrm{~min}$ for $\mathrm{Cr}$, while the locally furthest departure is found at $t=11 \mathrm{~h} 00 \mathrm{~min}$ for $\mathrm{Al}$ and $t=11 \mathrm{~h} 32 \mathrm{~min}$ for Cr. The composition of this particle crosses the volume-averaged particle composition (gray curves) once when viewed as a function of time, and twice when viewed as a function of particle size.

Particle 5 (cyan curves in Figs. 8c and 8d) experiences two coalescence events at $t=1 \mathrm{~h} 16 \mathrm{~min}$ and $t=2 \mathrm{~h} 56 \mathrm{~min}$, respectively. The earlier event does not alter the composition significantly: Particle size changes by factor $\Delta R=1.28$, and composition by factors $\Delta x_{\mathrm{Al}}=0.94$ and $\Delta x_{\mathrm{Cr}}=0.92$, the later event changes particle size by a factor $\Delta R=1.18$ and composition by $\Delta x_{\mathrm{Al}}=1.16$ and $\Delta x_{\mathrm{Cr}}=$ 
1.28. The composition of particle 5 also show multiple extrema: For Al, a locally closest approach to the equilibrium value is found at $t=3 \mathrm{~h} 36 \mathrm{~min}$, and a locally farthest departure at $t=6 \mathrm{~h} 52 \mathrm{~min}$, while for $\mathrm{Cr}$, locally closest approaches to equilibrium are found at $t=2 \mathrm{~h} 32 \mathrm{~min}$ and $t=3 \mathrm{~h} 24 \mathrm{~min}$ (before and after the second coalescence event), and a locally furthest departure from equilibrium at $t=7 \mathrm{~h} 32 \mathrm{~min}$. The composition of this particle remain closer to equilibrium than the volume-averaged particle composition following its departure from this curve at the first coalescence event, but crosses the volume-averaged particle composition once when viewed as a function of particle size.

\section{Discussion}

Extensive experimental investigations of phase separation in comparable AlCr-Ni alloys have been carried out with atom probe tomography (APT) e.g. by Mao et al. [6], and by Booth-Morrison et al. [22, 7]. Figure 3 in the latter publication shows $\gamma^{\prime}$ volume fraction, particle density, and average $\gamma^{\prime}$ particle radius for the comparable alloy $6.5 \% \mathrm{Al}-9.5 \% \mathrm{Cr}-\mathrm{Ni}$ aged at $873 \mathrm{~K}$, which may be compared with Figs. 3, 4a, and 5a in the present work on which we shall base this discussion.

Discounting the regime dominated by nucleation, both the $\gamma^{\prime}$ volume fraction, and the particle density are qualitatively similar. For the particle density, the simulations qualitatively correctly predict the rapid early decrease, the intermediate slowdown, and the final $n \propto t^{-1}$ phase. As the early decrease in particle density in the simulations was found to be due to coalescence, this is taken as evidence that coalescence is an important mechanism for microstructural evolution in the early stages of phase separation in this $\gamma-\gamma^{\prime} \mathrm{Al}-\mathrm{Cr}-\mathrm{Ni}$ alloy. Earlier studies have consistently found a high probability for $\gamma^{\prime}$ particles to form anti-phase boundaries rather than coalescing upon contact, dependent on their order parameter mismatch, which would reduce the importance of coalescence as a mechanism for microstructural evolution. However, Mao et al. [5] have shown that small $\gamma^{\prime}$ particles in proximity become interconnected by 
$\mathrm{L} 1_{2}$ ordered "necks" which facilitate coalescence for contacting particles. This phase separation in these $\gamma-\gamma^{\prime}$ alloys.

The intermediate slowdown was found to be characterized by decreased coalescence, and an absence of particle dissolution events as growth into the supersaturated matrix had resulted in a PSD without a tail of small particles that could readily dissolve into the matrix, before reaching the well-known classical coarsening regime. While the particle density becomes nearly constant in the simulations, a slight decrease is seen in the equivalent regime in the experimental data. This discrepancy is likely due to the initial conditions applied in the simulations: We introduce a random spatial arrangement of identical particles system is known to be a continuous process resulting in nuclei which may feature a continuum of sizes. So it is likely that experimentally determined PSDs in this transient regime would be skewed towards larger sizes, but still feature small (likely the most recently nucleated) particles, which may dissolve without a long delay required for the reconfiguration of the PSD towards smaller particle sizes, as was the case in the simulations.

It is more difficult to find the correspondence between the simulated and the experimental average particle radius: The simulated average particle radius increases, becomes constant and even slightly negative as growth and coalescence 495 cease, before finally increasing following the well-known $\langle R\rangle \propto t^{1 / 3}$ dynamics, while the experimental result appears to be increasing monotonically. This may possibly again be explained by the initial conditions of the simulations: The random spatial arrangement of nuclei results in relatively easily delineated growth and coarsening regimes, however if nucleation in the experimental alloy shows a tendency towards clustering, i.e. spatially preferred nucleation sites, the result might be microstructural evolution that is simultaneously dominated by growth at some spatial locations, and by coarsening at other spatial locations. Thus, a coarsening regime as was observed in the simulations where mass is transported from smaller particles to larger particles with few particle dissolution events 
may be occurring in the experimental system, but be masked by an increasing average particle radius due to concurrent growth at other spatial locations.

However, there is a marked discrepancy between the time scales of the experiment and the simulations, e.g. the $n \propto t^{-1}$ regime sets in around $t \approx 22 \mathrm{~h} 37 \mathrm{~min}$ in the simulations, while it seems to set in no earlier than $t \approx 256 \mathrm{~h}$ in the experiment. Contrary to the previous discrepancies between simulation and experiment, this is not believed to be related to the initial conditions for the simulations, but rather to the atomic mobilities employed in the simulations. Firstly, the atomic mobility matrices employed in the simulations are diagonal in the lattice-fixed frame of reference, which means that they do not admit correlations between the diffusive fluxes of different species, which is argued to be an important factor in the kinetic pathway for early-stage phase separation for comparable alloys [6]. As such, we would expect the system to choose quantitatively different pathways during phase separation, but it is not clear that inclusion of the diffusional flux couplings would result in microstructural evolution retarded by at least an order of magnitude. However, in the phasefield model applied in the current work, the phase transformation is required to be volume conserving, and thus the atomic mobilities must be transformed from the lattice-fixed frame of reference to the volume-fixed frame of reference [21], which therefore assumes that the vacancy distribution remains at equilibrium, or equivalently assumes a dense concentration of vacancy sinks and sources, i.e. dislocations, since the coherent particle-matrix interfaces do not act as sinks or sources for vacancies. For the range of particle densities reported by Booth-Morrison et al. [7] of $n=9.84 \times 10^{23}-3 \times 10^{21} \mathrm{~m}^{-3}$ we estimate a center-to-center interparticle distance via the Wigner-Seitz radius to be $d_{\mathrm{p}-\mathrm{p}} \approx 2(3 / 4 \pi n)^{1 / 3}=12-86 \mathrm{~nm}$, while the average distance between dislocations with a density of $\rho=10^{12}-10^{10} \mathrm{~m}^{-2}$ (typical values for well-annealed metals) is estimated as $d_{\mathrm{d}-\mathrm{d}} \approx \sqrt{1 / 2 \rho}=0.7-7 \mu \mathrm{m}$ [33], and so typical diffusional interactions (particle-matrix or particle-particle) in those experiments occur "far" from the dislocations required to equilibrate the vacancy concentra535 tion, which may thus explain the violation of the assumption of equilibrium of 
the vacancy concentration.

Another possible explanation for the difference between the time scales of the experiment and the simulations are unphysically large diffusivites caused by the uncertainty in the assessment of the atomic mobilities and the uncertainty in the Hessian matrix of the CALPHAD-optimized free energy functions. This is not believed to be the case, as all employed assessments are based on multiple experiments, however, for completeness, diffusion matrices and diffusion eigenmodes for the matrix phase which dominate the long-range diffusive behavior of the system are presented for inspection in the appendix.

Considerable progress in the understanding of the phase separation behavior in Al-Cr-Ni alloys has been made by Lattice Kinetic Monte Carlo (LKMC) simulations coupled with interaction energies from density functional theory $[4$, $5,6]$. LKMC is an atomistic method allowing simulations of vacancy-mediated diffusion in coherent phases, conserving the number of vacancies. However, the high computational demands for simulations with a realistically low vacancy concentration currently limits the time scales which may be probed, and so alternative simulation frameworks are desirable. To the best of the authors knowledge, no phase-field model has been presented to incorporate time-evolving vacancy distributions. Developing such a model would likely present a significant challenge, but would enable extension of quantitative phase-field methods into near-atomic length scales.

Finally, there are several different schemes for incorporating nucleation events into phase-field simulations, see e.g. [34] and references therein, however doing so at diffusive time scales in a computationally efficient, physically consistent manner must of this writing be considered challenging [35].

\section{Conclusions}

We have performed simulations of $\gamma-\gamma^{\prime}$ phase separation in $5.2 \% \mathrm{Al}-14.2 \% \mathrm{Cr}-$ $\mathrm{Ni}$ at $873.15 \mathrm{~K}$ with a phase-field model where the free energy functions were given by CALPHAD expressions [16], and the atomic mobilities were given 

functions and atomic mobilities were considered experimentally validated. Microstructural evolution was simulated up to a time of $32 \mathrm{~h}$ for 510 individual $\gamma^{\prime}$ particles corresponding to a $\gamma^{\prime}$ particle density of $10^{24} \mathrm{~m}^{-3}$ introduced as a spatially random distribution of identical particles chosen in accordance with classical nucleation theory.

The microstructural evolution was broadly characterized as consisting of 1) a regime dominated by growth and particle coalescence events, 2) growth with few particle coalescence events, 3) coarsening without particle dissolution events, and finally 4) "classical" coarsening, i.e. coarsening including particle $K$ agreeing well with the theoretical prediction by Morral and Purdy [31].

The microstructural evolution was found to agree qualitatively well with experimental evidence [7], but with considerable quantitative differences. This was argued to likely be due to the violation of the common assumption of the

inated by coarsening. The compositional trajectories of individual $\gamma^{\prime}$ particles were found to depart significantly from the volume-averaged composition when particle interactions and competition for supersaturation sets in, and showed complexities inherent to non-binary alloys.

\section{Acknowledgements}

The authors gratefully acknowledge economic support from DARPA grant W91CRB1010004, under which part of the work was performed, and SOP additionally gratefully acknowledges economic support from the Carlsberg Founda- 
tion. The authors thank Dr. Thomas Philippe for providing comments on the manuscript.

\section{Appendices}

$\gamma$ diffusivity

In this section, we present diffusivities of the $\gamma$ matrix phase which is expected to dominate the long-range diffusive behavior. The diffusivity matrices are given by the product of the chemical mobilities matrix in the volume-fixed frame of reference, and the Hessian matrix of the free energy density. Both are non-diagonal, and fully dependent on composition. The basis is (Al, Cr).

At the alloy composition $\vec{x}_{\text {alloy }}=(0.052,0.142)$ we have

$$
D_{\text {alloy }}=\left[\begin{array}{cc}
24.6 & 11.8 \\
11.8 & 9.43
\end{array}\right] \times 10^{-21} \mathrm{~m}^{2} / \mathrm{s}
$$

with corresponding fast and slow diffusion eigenvalue and vector pairs

$$
\begin{gathered}
\lambda_{\text {alloy }}^{\text {fast }}=31.0 \times 10^{-21} \mathrm{~m}^{2} / \mathrm{s}, \quad \vec{V}_{\text {alloy }}^{\text {fast }}=(0.878,0.479) \\
\lambda_{\text {alloy }}^{\text {slow }}=3.00 \times 10^{-21} \mathrm{~m}^{2} / \mathrm{s}, \quad \vec{V}_{\text {alloy }}^{\text {slow }}=(-0.479,0.878)
\end{gathered}
$$

While for the equilibrium (tie line) composition $\vec{x}_{\mathrm{eq}}=(0.036,0.150)$ we have

$$
\begin{gathered}
D_{\text {eq }}=\left[\begin{array}{rr}
16.3 & 6.74 \\
9.22 & 7.59
\end{array}\right] \times 10^{-21} \mathrm{~m}^{2} / \mathrm{s} \\
\lambda_{\text {eq }}^{\text {fast }}=21.0 \times 10^{-21} \mathrm{~m}^{2} / \mathrm{s}, \quad \vec{V}_{\text {eq }}^{\text {fast }}=\left(\begin{array}{ll}
0.823,0.568) \\
\lambda_{\text {eq }}^{\text {slow }}=3.00 \times 10^{-21} \mathrm{~m}^{2} / \mathrm{s}, \quad \vec{V}_{\text {eq }}^{\text {slow }}=(-0.450,0.893)
\end{array}\right.
\end{gathered}
$$

So $\lambda_{\text {alloy }}^{\text {fast }} / \lambda_{\text {eq }}^{\text {fast }}=1.48$ and $\lambda_{\text {alloy }}^{\text {slow }} / \lambda_{\text {eq }}^{\text {slow }}=1.00$. The fast modes align the fluxes of $\mathrm{Al}$ and $\mathrm{Cr}$, while the slow modes opposes the flux of $\mathrm{Al}$ and $\mathrm{Cr}$. 
The local phase compositions $\vec{x}_{\gamma}$ and $\vec{x}_{\gamma^{\prime}}$ and diffusion potentials $\tilde{\mu}_{\mathrm{Al}}$ and $\tilde{\mu}_{\mathrm{Cr}}$ must be determined in all interfacial regions at all times, as the evolution of the system depends on these quantities, as explained in Sec. 2. Due to the quasi-equilibrium assumption, these quantities are taken to be minimizers of the bulk free energy functional as given by Eq. (6).

If the current work had employed parabolic approximations to the free energies of the $\gamma$ and $\gamma^{\prime}$ phases, then Eq. (7) would reduce to a system of linear equations, which would thus be easily solved. However, as current work employs fully non-linear CALPHAD free energy functions, a more complex solution method is required, and Newton's method was chosen due to its fast convergence. Newton's method is an iterative method, where a vector of initial guesses is propagated towards an extremum by solving the system of linear equations

$$
\mathbf{H}\left[\vec{z}_{n+1}-\vec{z}_{n}\right]=-\lambda \nabla \overrightarrow{f_{\mathrm{bulk}}}
$$

where $\mathbf{H}$ is the Hessian matrix of $f_{\text {bulk }}, \lambda$ is a relaxation parameter to ensure convergence ( $\lambda=0.25$ in current work) and $\vec{z}$ is the vector of parameters to be determined, here chosen as $\vec{z}=\left(x_{\mathrm{Al}}^{\gamma}, x_{\mathrm{Cr}}^{\gamma}, x_{\mathrm{Al}}^{\gamma^{\prime}}, x_{\mathrm{Cr}}^{\gamma^{\prime}}, \tilde{\mu}_{\mathrm{Al}}, \tilde{\mu}_{\mathrm{Cr}}\right)$. This system of linear equations are transformed into triangular form by Gauss-Jordan elimination, and solved by back substitution, and the process is repeated until convergence, here taken to be when the sum of the magnitude of the elements of $\vec{z}_{n+1}-\vec{z}_{n}$ becomes less than $10^{-5}$. The elements of the Hessian matrix $\mathbf{H}$ and gradient $\nabla f_{\text {bulk }}$ are evaluated numerically with high precision as described in Sec. 2.6 .

\section{References}

[1] R. C. Reed, The Superalloys: Fundamentals and Applications, Cambridge University Press, 2006.

[2] Committee on Integrated Computational Materials Engineering, National Research Council of the National Academies, Integrated Computational 
Materials Engineering: A Transformative Discipline for Improved Competitiveness and National Security, National Academies Press, Washington, D.C., 2008.

[3] J. A. Christodoulou, Integrated Computational Materials Engineering and Materials Genome Initiative: Accelerating Materials Innovation, Adv. Mater. Process. 171 (2013) 28-31.

[4] C. Pareige, F. Soisson, G. Martin, D. Blavette, Ordering and phase separation in Ni-Cr-Al: Monte Carlo simulations vs three-dimensional atom probe, Acta Mater. 47 (1999) 1889 - 1899.

[5] Z. Mao, C. K. Sudbrack, K. E. Yoon, G. Martin, D. N. Seidman, The mechanism of morphogenesis in a phase-separating concentrated multicomponent alloy, Nat. Mater. 6 (2007) 210-216.

[6] Z. Mao, C. Booth-Morrison, C. K. Sudbrack, G. Martin, D. N. Seidman, Kinetic pathways for phase separation: An atomic-scale study in $\mathrm{Ni}-\mathrm{Al}-\mathrm{Cr}$ alloys, Acta Mater. 60 (2012) 1871-1888.

[7] C. Booth-Morrison, Y. Zhou, R. D. Noebe, D. N. Seidman, On the nanometer scale phase separation of a low-supersaturation Ni-Al-Cr alloy, Philos. Mag. 90 (2010) 219-235.

[8] N. Provatas, K. Elder, Phase-field methods in materials science and engineering, Wiley-VCH, 2010.

[9] L. Q. Chen, Phase-field models for microstructure evolution, Annu. Rev. Mater. Res. 32 (2002) 113-140.

[10] N. Moelans, B. Blanpain, P. Wollants, An introduction to phase-field modeling of microstructure evolution, CALPHAD 32 (2008) 268-294.

[11] I. Steinbach, Phase-field models in materials science, Model. Simul. Mater. Sc. 17 (2009). 
[12] N. Moelans, A quantitative and thermodynamically consistent phase-field interpolation function for multi-phase systems, Acta Mater. 59 (2011) 1077-1086.

[13] J. Eiken, B. Böttger, I. Steinbach, Multiphase-field approach for multicomponent alloys with extrapolation scheme for numerical application, Phys. Rev. E 73 (2006).

[14] M. Hillert, Some viewpoints on the use of a computer for calculating phasediagrams, Physica B \& C 103 (1981) 31-40.

[15] S. G. Kim, A phase-field model with antitrapping current for multicomponent alloys with arbitrary thermodynamic properties, Acta Mater. 55 (2007) 4391-4399.

[16] N. Saunders, Phase Diagram Calculations for Ni-based Superalloys, Proc. 8th Int'l Symp Superalloys (1996) 31-40.

[17] B. Sundman, J. Ågren, A regular solution model for phases with several components and sub-lattices suitable for computer applications, J. Phys. Chem. Solids 42 (1981) 297-301.

[18] L. Zhang, M. Stratmann, Y. Du, B. Sundman, I. Steinbach, Incorporating the CALPHAD sublattice approach of ordering into the phase-field model with finite interface dissipation, Acta Mater. 88 (2015) 156 - 169.

[19] A. Engström, J. Ågren, Assessment of diffusional mobilities in face-centered cubic Ni-Cr-Al alloys, Z. Metallkd. 87 (1996) 92-97.

[20] C. E. Campbell, Assessment of the diffusion mobilites in the gamma ' and B2 phases in the Ni-Al-Cr system, Acta Mater. 56 (2008) 4277-4290.

[21] J. O. Andersson, J. Ågren, Models for numerical treatment of multicomponent diffusion in simple phases, J. Appl. Phys. 72 (1992) 1350-1355. 
[31] J. E. Morral, G. R. Purdy, Particle coarsening in binary and multicomponent alloys, Scripta Metall. Mater. 30 (1994) 905-908.

[32] T. Philippe, P. W. Voorhees, Ostwald ripening in multicomponent alloys, Acta Mater. 61 (2013) 4237-4244. 
[33] D. Hull, D. Bacon, Introduction to Dislocations, 5th Ed., International series on materials science and technology, Elsevier Science \& Technology Books, 2011.

[34] T. W. Heo, L. Q. Chen, Phase-Field Modeling of Nucleation in Solid-State Phase Transformations, JOM 66 (2014) 1520-1528.

[35] J. Ågren, Nucleation - A Challenge in the Modelling of Phase Transformations, Proc. Int'l Conf. on Solid-Solid Phase Transformations in Inorganic Materials (PTM2015) (2015) 9-14. 